\title{
Innovative Ideas for the Teaching of Guzheng in Colleges and Universities based on the New Curriculum Reform
}

\author{
Xiaojuan Fu* \\ Suihua University, Suihua 152000, Heilongjiang Provience, China \\ *Corresponding author: Xiaojuan Fu, $459379261 @$ qq.com
}

\begin{abstract}
As a traditional musical instrument, the guzheng holds a very important status in the Chinese traditional culture. In recent years, with the increase of opportunities for exchanges and cooperation between the West and China, guzheng is rapidly favored in the foreign music circle. As the main institute for cultivating high-level talents in the society, the academic discipline of music in various universities should actively promote the education reform of guzheng based on the new curriculum reform combined with innovations and modifications of the national instrumental music teaching model to efficiently teach guzheng.
\end{abstract}

Keywords: New curriculum reform; Colleges and universities; Guzheng teaching; Innovation path

Publication date: June 2021; Online publication: June 30, 2021

\section{Introduction}

Guzheng is the most typical among the classical musical instruments in China which has been passed down for nearly two thousand years. In recent years, with the integration of Chinese culture with the world culture, the art of guzheng has gradually been accepted and highly sought after by the Westerners. Hence, colleges and universities should follow the development trends of guzheng and continuously innovate teaching methods in the education of this musical instrument. Based on the analysis of issues related to the teaching of guzheng under the new curriculum reform, this article provides an in-depth discussion about new innovative ideas in the teaching of guzheng in hope to cultivate more highly skilled guzheng professionals.

2. Problems related to the teaching of guzheng in colleges and universities based on the new curriculum reform

\subsection{Inadequate sense of innovation among guzheng teachers}

In regard to the present situation of guzheng education in local colleges and universities, many of the problems are due to the lack of innovative skills among teachers. The lack of innovative consciousness in many guzheng teachers is attributed to two reasons. Firstly, the education is realized by traditional teaching methods in which the traditional method neglects the cultivation of innovative consciousness. Teachers are accustomed to use traditional teaching methods and in view of short class periods, time does not permit innovation. Secondly, many colleges and universities that offer guzheng trainings often only focus on improving students' performances, but they completely neglect the students' future development in which it is understood that students' excellent performances are signs of their future development. It is often believed that the students only need to have skills to play the guzheng in their future work; hence, disregarding the improvement of their comprehensive abilities. In this way, teachers subjectively tend to emphasize on academic performances but neglect the innovation of guzheng teaching. ${ }^{[1]}$ 


\subsection{Single teaching form in the guzheng course}

For a long time, in regard to the teaching of guzheng, many local colleges and universities have been using the same teaching plan without any new content ideas. They also reject advanced teaching concepts; hence it is difficult to keep up with the development pace over time. The form of teaching is also utterly derailed from the development needs. A single teaching form cannot completely stimulate students' enthusiasm for learning which results in substandard teaching qualities.

\subsection{Emphasis on skill training while neglecting the systematic learning of modern guzheng music}

At present, many colleges and universities have been emphasizing on skills training in regard to playing the guzheng but they neglect the systematic learning of modern guzheng repertoires. In the education of guzheng, playing skills are certainly important but teachers should also understand that the art and culture of guzheng needs to be inherited, and moreover, innovation is necessary. Only the education which can adapt to the development trends would persevere. In the context of the new curriculum reform, many teachers do not emphasize on these issues. They are unable to accept or keep up with new trends, hence the effects of teaching are unable to reach the expected goals. ${ }^{[2]}$

\subsection{Hindering individual development with the neglect of students' dominant status in classrooms}

The traditional teaching model is a fixed model where teachers teach or tutor while students just attend and learn. Especially in the teaching of guzheng, this model is particularly obvious. Just like "the master leads the apprentice," teachers would dominate the entire classroom and maintain their authority. This model significantly inhibits students' individual development. Over time, students' enthusiasm for learning will gradually deplete and at the same time, their inspirations for guzheng playing would be inhibited.

\section{Innovative ideas of guzheng teaching in colleges and universities under the new curriculum reform}

\subsection{Clarifying teaching goals and carrying out teaching designs}

In the innovation of guzheng teaching, it is first necessary to clarify that the new curriculum reform would improve the teaching of guzheng in colleges and universities. Then, it is necessary to propose the teaching goals under the new curriculum reform while combining them in formulating systematic teaching plans. It is a necessity for the reform of guzheng teaching to be guided by fundamental goals of the new curriculum reform while continuously enhancing students' practical skills and cultivating them to be compounding talents that meet the development needs over time. At the same time, it is also necessary to comprehensively consider the actual situation of colleges and universities while formulating new curriculum reform teaching goals that are suitable for the development of these institutes. The goal reform can be divided into three levels which are basic goals, practical goals, and expanded goals. ${ }^{[3]}$

\subsubsection{Basic goals}

Guzheng teachers should emphasize on the basic teaching of guzheng including basic knowledge and basic playing skills. They should provide students with more practical opportunities so that students may learn and sublimate basic knowledge in practice, hence mastering a solid theoretical foundation and having excellent playing skills.

\subsubsection{Application goals}

Teachers and schools should meet the requirements of the society in the development of guzheng, constantly adjust their teaching goals and devote themselves to the students' future development in order 
to develop the cause of Guzheng. At the same time, they should also conduct targeted trainings in accordance with the future development trends of students and provide assistance to direct students in developing career plans and eventually, choosing their own careers. For example, for students who have the interest to engage in guzheng performances in the future, the school as well as the teachers should arrange more opportunities for performances and objectively evaluate them after the performance. This would encourage students to accumulate experiences, gain confidence, and gradually improve their performance skills to progress to a more advanced stage.

\subsubsection{Expansion goals}

On the other hand, there would be students that would not choose to venture into the professional guzheng career in the future but instead, they would choose to work in professions related to this field. For these students, although their guzheng knowledge and ability requirements are not as excellent as those of the professional guzheng performance students, they would still have a certain degree of guzheng performance skills. This requires teachers, colleges, and universities to modify their thought process during teaching in which the cultivation of students' compound skills, professional qualities, and sense of cooperation should also be emphasized in order to gain more employment opportunities. It can be seen from this that under the new curriculum reform, college teachers and schools should adhere to student-oriented approach in their daily teaching practices and focus on targeted guzheng education based on the actual state of the students and the school as well as their future development in order to cultivate highly qualified talents in the society.

\subsection{Highlighting the status of students}

The new curriculum reform requires that contemporary teachers should highlight the student's status when conducting lessons, establish a relationship with students, constantly stimulate their subjective learning consciousness, and completely change the traditional teaching method in which students passively learn. This is especially true in the guzheng teaching. Teachers should thoroughly analyze the importance of learning the guzheng while improving students' understanding of the instrument from various perspectives and for them to acknowledge the advantages of Guzheng. Due to the deep-rooted influence of the traditional teaching method, many guzheng teachers in colleges and universities have not corrected their teaching methods. They still conduct lessons with unilateral "output," hence the effects of teaching are unable to meet the expected outcomes. With the vision of the new curriculum reform, teachers need to first, recognize the teaching goals of this new curriculum reform, transform their identities, swap the roles in classrooms, participate in students' learning as friends, listen carefully to students' inner thoughts, and guide them systematically. This method of learning helps students to master their skills in guzheng at a faster pace and transforms the classroom into a warm, relaxed, pleasant, and self-inquiring learning environment. For example, when teaching the song, "The Night among Fishing Boats," teachers may start off by using the multimedia to explain the background of the song so that students would be able to understand the creative intention of the song and feel its creative mood before playing it. In this way, not only their interest in learning would be significantly stimulated but they would also be able to truly experience the joy of learning guzheng which would continuously enhance their interest in learning. ${ }^{[4]}$ Throughout this process, teachers should also explain and demonstrate some difficult skills in the performance of the repertoire so that students would be able to achieve the goal of "practice makes perfect" after repeated training. Due to different levels of professional qualities and receptive skills among students, teachers should appreciate their differences, conduct hierarchical teaching as a respect to individual differences, actively answer students' questions, and improve classroom teaching efficiency. 


\subsection{Innovating classroom teaching methods and strengthening practical teaching}

\subsubsection{Innovative classroom teaching methods}

Guzheng inherits the music culture of China of nearly two thousand years and shows the charm of ancient Chinese culture. In order to for the teaching of guzheng to be more efficient, contemporary teachers and universities should fully understand the characteristics of the education under the new curriculum reform whereby teaching has been modernized and improved. Traditional guzheng teaching methods often only focus on the cultivation of students' basic knowledge and playing skills while neglecting their future development. Hence, students lack comprehensiveness in their future career planning. Therefore, to achieve innovations in the teaching methods of guzheng, the first step has to be based on student-oriented teaching in which the teaching goals should be in regard to students' development. In classrooms, teachers should combine modern teaching aids with diversified teaching methods by making full use of network resource collection and sharing capabilities. Teachers may share their guzheng playing skills and upload the latest Guzheng repertoires onto sharing platforms so that students would be able to learn guzheng at any time even during their spare time. In addition to that, teachers should consciously surrender their dominant status in classrooms and start to make friends with their students to enhance teacher-student interactions and narrow the teacher-students distance while creating a bright and relaxed environment for learning. For example, before classroom teaching begins, teachers should encourage students to use the internet to search for the background of the repertoires that would be taught so that students may understand them in advance. During classes, teachers should also use modern teaching methods and tools to display the charm of guzheng. In these ways, the interests of students in learning guzheng would be stimulated by their intuitive feeling of music and emotional experiences in order to achieve the purpose of improving the quality of teaching. ${ }^{[5]}$

\subsubsection{Providing more practical learning opportunities to enhance students' practical application skills}

In order to achieve high-quality teaching of guzheng under the new curriculum reform, teachers need to actively innovate and break the constraints of time and space in the traditional teaching model. Specifically, in the guzheng teaching reform, teachers should refer to successful teaching reform experiences of other colleges and universities, then combine the specific conditions of their schools to reform teaching contents, modes, philosophies, and methods while focusing on students' basic knowledge, skills, and performances. While cultivating technical skills, emphasis should be on the improvement of students' practical application skills. Schools may allocate studios for students majoring in guzheng and encourage them to conduct guzheng performances or perform in meaningful public welfare activities. By actively encouraging them to participate in these performances, they would be able to accumulate stage experiences, improve in organizing performances, and build up their cooperative skills. If condition permits, schools can also cooperate with enterprises or commercial performance agencies to cultivate guzheng talents that are needed by the society. ${ }^{[6]}$

\section{Conclusion}

For teachers and students in the guzheng major, it is necessary for them to continuously accumulate profound cultural background and conduct studies or research on guzheng based on rich knowledge and science. When teaching guzheng, teachers are not only required to fully understand the unique style and charm of guzheng but also to explain the artistic performance. When learning Guzheng, students should not only learn the related theoretical knowledge of guzheng music but also focus on the improvement of cultural accomplishment which is the basis for learning this skill. In order to truly improve the quality of 
guzheng teaching, teachers and schools should effectively combine the unique style of guzheng with national music in order to realize the inheritance and development of guzheng as well as to achieve the goal of inheriting classical consciousness.

\section{Disclosure statement}

The author declares no conflict of interest.

\section{References}

[1] Yang L, 2020, Discussion on the innovative ideas of guzheng teaching in colleges and universities from the perspective of the new curriculum reform. Drama House.

[2] Yin D, 2019, Research on the innovation path of guzheng teaching in colleges and universities from the perspective of new curriculum reform. Contemporary Educational Practice and Teaching Research.

[3] Li L, 2018, Research on the innovative path of guzheng teaching in colleges and universities under the new curriculum reform. Art Education.

[4] Sha M, 2016, On the cultivation of inner hearing in college Guzheng teaching. Journal of Yunnan University of the Arts.

[5] Zhang W, Tan Q, Hu Y, 2015, The application of guqin performance in chamber music works from the "Guqin Ensemble". Shanghai Conservatory of Music.

[6] Wu H, Lu B, Sun Z, 2017, The inheritance, innovation, and performance characteristics of Zheng music. Nanjing University of the Arts.

[7] Jia X, Hu Y, Qin S, 2014, Analyzing the aesthetic thought of guqin music in "Qin Jue" and its perspective on contemporary music practice. China Conservatory of Music. 